\title{
Mouvement agroécologique au Brésil : trajectoire, contradictions et perspectives*
}

\author{
Alfio Brandenburg \\ Sociologue, Université fédérale de l'État de Paraná, Département Sciences sociales, 460 rue General Carneiro, 80060-150 Curitiba, \\ Paraná, Brasil
}

\author{
Mots-clés : \\ mouvement \\ écologique ; \\ agriculture ; \\ agriculteurs \\ écologiques ; \\ nouveaux acteurs ; \\ Brésil
}

\section{Keywords:}

ecological movement; agriculture; organic farmers; new actors; Brazil

\begin{abstract}
Résumé - Le présent texte analyse la trajectoire du mouvement alternatif ou écologique dans l'agriculture, en prenant comme référence des données et des informations provenant d'études réalisées au Brésil. Cette analyse privilégie les aspects liés à l'origine, à l'expansion et à l'institutionnalisation de l'agriculture écologique. Le nombre d'agriculteurs qui pratiquent l'agriculture écologique est donné pour chacun des États de la fédération en distinguant les catégories d'agriculteurs, les différentes formes d'agriculture écologique, ainsi que les stratégies d'organisation des agriculteurs et de commercialisation de la production, et en précisant les organismes d'appui et d'orientation technique de même que la réglementation de la pratique écologique et de ses implications. La conclusion comporte une brève réflexion sur des questions liées aux conséquences du processus d'institutionnalisation et aux perspectives de l'agriculture écologique.
\end{abstract}

\begin{abstract}
The Agroecological movement in Brazil, its trajectory, contradictions and perspectives. Our paper analyzes the trajectory of the alternative or ecological movement in agriculture based on data and information from studies carried out in Brazil. Our analysis privileges aspects relating to the origin, expansion and institutionalization of ecological agriculture. In dealing with these different moments, we considered the following data and phenomena: number of farmers who practice ecological agriculture by state; types of farmers; different forms of ecological agriculture; farmers' organizational strategies and strategies for getting their produce on the market; services providing support and technical information and regulations applying to ecological farming and their implications. We conclude with a brief reflection on several questions regarding the consequences of the institutionalization process and prospects for alternative agriculture.
\end{abstract}

\section{Introduction}

Au Brésil, le mouvement de l'agriculture écologique a commencé avec le mouvement d'opposition à une agriculture moderne dont la logique était l'exclusion sociale de milliers de petits agriculteurs. Cependant, le mouvement ne s'est pas limité à contester le modèle de la modernisation, il a aussi proposé des pratiques sociales et des techniques qui se sont imposées avec le temps comme des modèles pour une agriculture alternative à l'agriculture moderne d'exportation. À cause de ses

Auteur correspondant : alfio@onda.com.br

A. Brandenburg enseigne la sociologie (sociologie rurale, sociologie de l'environnement) dans le cadre des formations doctorales en sociologie et en environnement et développement de l'Université fédérale de l'État de Paraná.

* Traduction : Nathalie Dessartre techniques de production, les chercheurs du domaine agraire considéraient ce mouvement comme passéiste, dépassé et, partant, inintéressant.

Ces dernières années, avec l'aggravation des problèmes environnementaux et des risques sociaux de la technologie, le mouvement écologique gagne du terrain. Ainsi, l'agroécologie est considérée comme une des formes d'inclusion sociale des familles des petits agriculteurs. Toutefois, parallèlement à l'augmentation de la demande en produits naturels, l'agriculture écologique cesse peu à peu de relever exclusivement de la responsabilité des agriculteurs, pour être également pratiquée par d'autres catégories professionnelles qui agissent parallèlement comme militants, encourageant les pratiques écologiques et conduisant ces mouvements sociaux.

Face à l'expansion du mouvement et à la participation de diverses catégories sociales, quelques questions 
se posent : peut-on considérer l'agriculture écologique comme une pratique de préservation de l'environnement et, simultanément, d'inclusion sociale des agriculteurs exclus de la modernisation? Quelles sont les perspectives pour les produits écologiques? Ont-ils les moyens de concurrencer les produits conventionnels sur le marché? Quelles sont les conséquences de l'entrée de ces produits dans la compétition commerciale et pour le mouvement écologique? Nous tenterons, ici, d'analyser ces questions en prenant comme fil conducteur la trajectoire du mouvement agroécologique, dont nous prenons en considération trois phases : sa genèse, son expansion et son institutionnalisation ${ }^{1}$. Pour la première phase, nous analyserons les conditions d'émergence du mouvement agroécologique, ainsi que sa spécificité face aux autres mouvements mondialement connus. Concernant la phase de l'expansion, nous montrerons comment ce mouvement s'organise et s'étend dans les divers États brésiliens. Enfin, pour la phase de l'institutionnalisation, nous analyserons les politiques d'encouragement à la production, les implications des normes qui réglementent l'agriculture écologique et l'adaptation des produits écologiques aux exigences du marché.

\section{Agriculture alternative : l'origine du mouvement}

Au Brésil, en tant que mouvement socialement organisé, l'agriculture alternative est née dans les années 1970. Il s'agissait d'un contre-mouvement opposé à la politique de modernisation. Ce mouvement était la conséquence d'une politique agricole qui ne permettait pas aux petits agriculteurs, voués à une culture de subsistance, de bénéficier du crédit agricole de l'infrastructure et des services d'orientation technique. Ainsi, l'agriculture alternative, qui est apparue initialement comme un mouvement de contestation et de proposition de pratiques écologiques, s'est transformée en un modèle alternatif à l'agriculture industrielle capitaliste, connue aujourd'hui sous le nom d'agromarché.

Cependant, le mouvement alternatif brésilien n'est pas le seul dans l'histoire de l'agriculture. Avant même que ne soient perçues les conséquences de ce que l'on a appelé la révolution verte, dans la première moitié du $X X^{\mathrm{e}}$ siècle, naquirent en Europe, et même aux États-Unis, des mouvements semblables ${ }^{2}$. Au Brésil, il n'existe pas

\footnotetext{
${ }^{1}$ Ce texte est produit avec l'appui d'une bourse CAPES (Coordenação de Apoio ao Ensino Superior [Coordination d'appui à l'enseignement supérieur]). Nous remercions Hugues Lamarche, Jean-Paul Billaud et Götz Schmidt pour nous avoir servi d'interlocuteurs.

2 En Allemagne, en 1924, ce fut l' « agriculture biodynamique et naturelle» (Vogt, 2000), en Angleterre, en 1946, l'« agriculture biologique » (Reed, 2001) et en France, en 1940, 1'« agriculture biodynamique d'alimentation normale» (César,1999).
}

d'informations bibliographiques au sujet d'un mouvement analogue à cette époque-là. Toutefois, les fondements pratiques pour établir une agriculture écologique existaient déjà avant la période de la modernisation. Les immigrants européens ont introduit des systèmes de production basés sur la gestion des ressources naturelles provenant de la première révolution agricole. Mais c'est à partir de ce que l'on appelle la modernisation conservatrice que les petits agriculteurs, menacés d'exclusion ou directement exclus par les mécanismes de l'expropriation de la politique agricole, s'organiseront. Privés de l'aide des services officiels, ces agriculteurs sont des militants des Églises catholique ou protestante. Les Commissions pastorales de la terre vont développer auprès des agriculteurs un travail qui, dans certains cas, va se faire par l'intermédiaire d'organes créés dans le but spécifique de les orienter. Du point de vue technique, ces Commissions pastorales de la terre réhabilitent des pratiques traditionnelles que les agriculteurs connaissent déjà, visant à élaborer un ensemble de stratégies susceptibles d'assurer la reproduction sociale des agriculteurs dans les campagnes. L'agriculture alternative représente un moyen de survie pour le petit agriculteur et signifie la reconstruction d'une relation socioenvironnementale qui trouve son origine dans la condition paysanne.

\section{Les différentes formes de l'agriculture écologique}

Par agriculture écologique, on entend l'agriculture qui englobe un ensemble de modèles alternatifs par rapport au modèle agro-industriel de production. Cette agriculture va des modèles qui sont à l'origine même du mouvement alternatif jusqu'à ceux auxquels on a attribué une nouvelle signification en fonction des mouvements écologiques récents et qui sont réglementés par les politiques agricoles.

L'expansion de l'agriculture écologique, qui accompagne également la valorisation contemporaine de l'écologie dans la société, est soutenue par des agriculteurs d'élite, par des néoruraux et par des professionnels qualifiés $^{3}$ (Karam, 2001 ; Balestrin, 2002). Cela ne signifie pas pour autant que les agriculteurs traditionnels ne participent pas au mouvement. Bien au contraire, au Brésil, on constate une augmentation du nombre d'agriculteurs traditionnels qui convertissent leurs unités conventionnelles de production agricole en unités de production écologiques. Autrement dit, l'agriculteur écologique ne constitue pas une catégorie sociale homogène. Partant, l'agriculture écologique n'étant pas une stratégie de reproduction d'une catégorie sociale spécifique, elle ne peut pas être considérée comme une solution aux problèmes

\footnotetext{
${ }^{3}$ Les professionnels qualifiés sont des techniciens spécialisés ou des agronomes installés qui pratiquent l'agriculture écologique.
} 
relatifs à la survie et à la reproduction d'une catégorie déterminée.

Parmi les agriculteurs écologiques, on peut donc identifier des petits agriculteurs traditionnels, des néoruraux, des petits entrepreneurs, des entreprises associatives, des coopératives de production, des entreprises qui produisent sous contrat. Ce sont des catégories sociales qui, d'une certaine façon, sont engagées, par l'intermédiaire de leurs associations, dans différents courants de la pensée agroécologique. Dans ce contexte, un courant de pensée écologique peut, par conséquent, englober diverses catégories, de même qu'une catégorie sociale peut prendre en compte différents courants. Les catégories sociales et les courants de la pensée écologique s'entrecroisent et forment un réseau d'interactions complexe. C'est pourquoi il n'est pas toujours possible, dans la pratique, d'identifier des agriculteurs dont l'idéologie remonte à la genèse du mouvement écologique. Le pragmatisme et l'expérience éloignent certains acteurs des principes idéologiques adoptés à l'origine du mouvement. Par contre, les militants ou les pionniers du mouvement constituent une exception.

Depuis le Sommet de Rio, en 1992, l'agriculture écologique a reçu l'appui des associations, des ONG et des associations publiques d'assistance technique, telles que l'EMATER $^{4}$, qui la nomme "agriculture soutenable». L'agriculture soutenable est donc comprise comme une forme d'organisation de production apte à valoriser au mieux les ressources et l'usage réduit d'intrants.

Face à l'expansion des mouvements écologiques et à une demande de produits agricoles présentant moins de risques pour la santé, l'agriculture soutenable trouve dans l'écologie une justification pour un nouvel essor et une redynamisation. L'agriculture écologique, représentée par différents courants (comme, par exemple, l'agriculture biologique, la biodynamie, l'agroécologie, la permaculture), trouve un soutien, en particulier, auprès $\mathrm{d}^{\prime}$ associations dirigées par des techniciens agronomes qui retournent à la campagne. Ils sont appelés les néoruraux ${ }^{5}$ (Karam, 2001).

Parmi les formes de tradition européenne, l'agriculture biologique est celle qui se développe le plus au Brésil, notamment chez les néoruraux et chez les chefs d'entreprises agricoles. L'expansion de ce modèle est due au fait qu'il s'agit d'une agriculture dont l'organisation productive ressemble à l'agriculture conventionnelle, surtout pour ce

\footnotetext{
${ }^{4}$ Organisation publique d'assistance technique et d'extension rurale.

${ }^{5}$ On appelle néoruraux les agriculteurs qui ont vécu dans un milieu urbain, mais qui sont retournés dans les campagnes, par l'intermédiaire du mouvement de contre-culture des années 1960 en Europe. Dans la région métropolitaine de Curitiba, 60 \% des agriculteurs écologiques proviennent de secteurs professionnels non agricoles, bien qu'ils aient des antécédents dans le milieu rural.
}

qui est de son degré de spécialisation. Le mode organique de production écologique se base sur l'apport à la terre d'une quantité élevée de matière organique dans le but de maintenir une activité intense de microorganismes responsables de la fertilité, facteur essentiel pour l'élimination des maladies des plantes. Depuis peu, grâce au cahier des charges, il est permis d'utiliser des engrais organiques ou biologiques qui remplacent les engrais chimiques.

L'agriculture biodynamique, pratiquée à une moindre échelle, se base sur l'influence des astres sur la vie des plantes, sur les composts biodynamiques, sur l'engrais vert, sur la culture de la biodiversité. Mais le principal objectif de ce mode d'agriculture consiste à transformer la propriété agricole en une sorte d'organisme vivant où les activités agricoles et d'élevage se complètent. Une autre manière de pratiquer l'agriculture qui inspire les agriculteurs écologiques est la permaculture. Ce système de production, d'origine australienne, cherche à construire des agroécosystèmes qui simulent les écosystèmes naturels, en incluant des espèces pérennes, notamment des arbres de taille moyenne à grande. L'approche de la permaculture est large, car elle inclut l'architecture de maisons et tout un habitat écologiquement durable.

Mais, parmi les divers systèmes de production, celui qui occupe le plus de terrain parmi les agriculteurs qui font partie des mouvements sociaux est l'agroécologie. Apparue en Amérique latine, l'agroécologie est non seulement une pratique agricole, mais aussi une pratique qui s'appuie sur des savoirs, des valeurs sociales, culturelles et politiques dans le but de construire des relations sociales et environnementales liées aux intérêts des paysans ou des petits agriculteurs. À la base de cette modalité d'agriculture écologique se trouve la certification participative des agriculteurs et leur organisation en réseau ${ }^{6}$.

Bien que l'on ne dispose pas de données précises sur l'évolution de chacune de ces modalités au niveau national, des évaluations indiquent l'avancée significative, sous la rubrique "agriculture biologique », de l'ensemble des formes de production mentionnées dans le tableau 1. Nombre des agriculteurs hors des circuits de l'aide technique officielle ou de celle d'associations ne sont pas pris en compte, par exemple les agriculteurs de assentamentos ${ }^{7}$, qui choisissent de plus en plus l'agriculture écologique comme base pour leur reproduction sociale.

On observe que, dans l'État du Paraná, se concentre le plus grand nombre d'agriculteurs ou de propriétés de cultures biologiques (4 122 sur un total de 12801 propriétés), tandis que le Mato Grosso compte la plus grande surface cultivée (80 258 ha, sur un total de 811519 ha). D'après des informations du ministère de l'Agriculture, ces données concernent divers produits : des fruits

\footnotetext{
${ }^{6}$ Dans le Sud du Brésil, les agriculteurs sont organisés dans le réseau Ecovida (Écovie) de l'agroécologie.

7 Terres cédées par la réforme agraire pour que les agriculteurs $\mathrm{s}^{\prime} \mathrm{y}$ installent et produisent.
} 
Tableau 1. Estimation du nombre de producteurs biologiques, des surfaces cultivées, du volume de production et des ventes au Brésil, en 2005.

\begin{tabular}{|c|c|c|c|c|}
\hline État fédéral & Nombre de propriétés & Surface (ha) & Volume de production (t) & Ventes (en millions de $\mathrm{R} \$$ ) \\
\hline Rio Grande do Sul & 1662 & 3716 & - & 40 \\
\hline Santa Catarina & 706 & 5922 & 17000 & 18 \\
\hline Paraná & 4122 & 11252 & 66256 & 50 \\
\hline São Paulo & 533 & 52210 & - & 70 \\
\hline Rio de Janeiro & 51 & 1485 & - & - \\
\hline Espírito Santo & 137 & 2606 & - & - \\
\hline Minas Gerais & 263 & 14286 & - & - \\
\hline Distrito Federal & 164 & 852 & & \\
\hline Goiás & 11 & 7030 & & \\
\hline Mato Grosso do Sul & 176 & 13440 & & \\
\hline Mato Grosso & 217 & 80258 & & \\
\hline Bahia & 431 & 8217 & & \\
\hline Sergipe & 92 & 817 & & \\
\hline Alagoas & 62 & 12366 & & \\
\hline Pernambuco & 255 & 1052 & & \\
\hline Rio Grande do Norte & 115 & 1788 & & \\
\hline Paraíba & 184 & 1857 & & \\
\hline Ceará & 151 & 16420 & - & - \\
\hline Piauí & 40 & 28619 & & \\
\hline Maranhão & 474 & 8175 & - & \\
\hline Pará & - & - & - & - \\
\hline Acre & 119 & 32379 & & - \\
\hline Amazonas & - & - & & \\
\hline Rondônia & 100 & 1656 & & \\
\hline Tocantins & 1 & 12100 & & \\
\hline Amapá & 106 & 4540 & & \\
\hline TOTAL & 12801 & 811519 & 300000 & R\$ 300 millions \\
\hline
\end{tabular}

Source : Ministério da Agricultura, Pecuária e Abastecimento.

(goyave, fraise, cerise, fruit de la passion, banane, raisin, citriques); des cultures maraîchères (salade, chou, tomate, carotte, cresson, aubergine); des cultures annuelles et pérennes (riz, soja, maïs, blé, manioc, café, cacao, canne à sucre); de la production animale (viande bovine et porcine, volaille, lait, œufs, poisson et miel) ; des productions d'extraction (cœur de palmier, noix du Brésil, noix de cajou, baie brésilienne, babaco). Les trois États du Sud du Brésil (Paraná, Santa Catarina et Rio Grande do Sul) sont les premiers pour le nombre d'agriculteurs écologiques. L'agriculture écologique y bénéficie d'un environnement favorable à sa mise en œuvre et à son expansion. Sans doute est-ce parce que s'y concentrent des immigrés européens : allemands, italiens et polonais.

Au Brésil, des organismes d'aide ont motivé les agriculteurs biologiques à s'organiser en associations autogérées, dans le but de construire un espace de discussion sur leurs conditions de vie et sur les problèmes de production et de commercialisation. Ces associations, avec les ONG, ont agi sous la forme de contrepouvoir face aux organismes gouvernementaux, en présentant des critiques contre les politiques de l'État.

La multiplication de groupes et d'associations engagés dans la quête de techniques alternatives de production a été favorisée par l'organisation d'une rencontre qui a eu lieu à Campinas (État de Saõ Paulo) en 1983, à laquelle ont participé plus de cent leaders nationaux, parmi lesquels des techniciens et des agriculteurs représentant les divers États du Brésil. C'est à ce moment qu'est né le Projeto Tecnologias Alternativas (Projet de technologies alternatives), auquel participent les dix États brésiliens suivants : Maranhão, Ceará, Paraíba, Pernambuco, Santa Catarina, Bahia, Paraná, São Paulo, Rio Grande do Sul et Minas Gerais (Weid, 1988).

Grâce à l'expansion du mouvement agroécologique, en particulier dans les années 1990, les associations et les ONG ont créé un forum de coordination nationale d'agroécologie, à partir de la première Rencontre nationale d'agroécologie en juillet 2002. Cependant, ce renforcement du mouvement écologique en tant que 
mouvement articulé en réseau ne s'est pas réalisé sans conflits à l'intérieur des organisations. La présence, à côté des agriculteurs, de professionnels de l'agriculture comme les conseillers agricoles au sein de ces organisations est à l'origine d'oppositions sur les modèles techniques appliqués par des agriculteurs et par des professionnels (Balestrin, 2001). Par exemple, dans le groupe analysé par Balestrin, en raison des divergences, une partie des membres organise une association pour la commercialisation des produits écologiques, et l'autre organise une entité d'appui aux agriculteurs. Toutefois, ces conflits n'ont pas qu'un aspect négatif ; ils permettent à chacun d'affirmer son identité. Cette identité, formée dans le mouvement social, constitue une dimension fondamentale de la reconnaissance professionnelle de chaque agriculteur, qui apparaît comme un nouvel acteur ${ }^{8}$ dans le contexte agricole brésilien.

\section{L'institutionnalisation de l'agriculture écologique}

À partir de leurs organisations, de leurs associations ou même individuellement, les agriculteurs ont construit leur stratégie de relation avec le marché. Pour garantir leur viabilité du point de vue économique, il leur fallait trouver d'autres voies, différenciées par rapport au marché de consommation de masse, afin de commercialiser leurs produits. Ainsi, ils ont privilégié le système de transaction commerciale directe avec le consommateur, où les contacts personnels favorisent la création d'une clientèle fidèle aux produits alternatifs. Les formes mises en place ont été les suivantes : marchés de produits alternatifs ou écologiques, systèmes de livraison directe au consommateur, événements spéciaux tels que fêtes et expositions de produits, vente dans la propre propriété agricole (Rucinski, 2000).

Les systèmes de commercialisation alternatifs ne jouent pas seulement le rôle de transaction commerciale, ils sont aussi des espaces de sociabilité, de dégustation de produits et de rééducation d'habitudes alimentaires (Rodrigues, 2001). En outre, à partir de ces espaces de sociabilité, se développent des liens de solidarité entre producteurs et consommateurs qui donnent lieu à des organisations de consommateurs de produits écologiques (Rucinski, 2000).

Si la construction d'un commerce alternatif s'est réalisée à la demande d'un consommateur de type idéologique, le développement du marché pour les produits écologiques, observé principalement à partir des années 1990, est dû à un autre type de consommateur: le «consommateur-acteur, ou critique». Ce dernier, influencé

\footnotetext{
${ }^{8}$ Nous définissons l'agriculteur écologique comme un nouvel acteur, car il reconstruit simultanément les pratiques concernant le rapport avec la nature et celles de l'organisation sociale.
}

par des sources d'informations diverses, parmi lesquelles les moyens de communication de masse, observe quelques critères tels que la présentation, le prix et la diversité des produits. Cependant, il se différencie du consommateur de masse, car ses choix portent sur des aliments sans résidus toxiques avec label de garantie (Silva, 2006). Obéissant aux normes, les écoproduits disputent l'espace de vente aux produits industriels comme les produits diététiques, hydroponiques, de qualité, etc. Bien que l'expansion du marché des produits écologiques se soit réalisée grâce au grand réseau des supermarchés (Guivant, 2003 ; Silva, 2006), les structures de commercialisation considérées comme alternatives existent toujours. C'est le cas, par exemple, des marchés municipaux locaux organisés par les agriculteurs, du système de livraison directe, du commerce spécialisé, etc.

Ce développement du marché de produits écologiques a abouti à une reconnaissance de l'identité de l'agriculteur écologique et à une réglementation de la production garantissant l'absence de résidus toxiques dans les aliments. Au Brésil, l'agriculture biologique a été réglementée pour la première fois en 1999, par le ministère de l'Agriculture. Des normes de production, de transformation, d'étiquetage des produits biologiques ont été établies, puis actualisées en 2003.

Avant la réglementation de la production, les organismes qui concédaient le label de qualité, tel l'Institut biodynamique (IBD), le plus connu, travaillaient déjà dans diverses régions du pays ${ }^{9}$. Bien que le label soit important du point de vue de l'identification du produit sur le marché, il commence à poser des problèmes à certains (Bye et Schmidt, 2001). Ne serait-il pas un facteur supplémentaire d'exclusion sociale? Dans ce cas, quelle serait la forme la plus adéquate d'insertion dans le marché? Y aurait-il des conditions permettant que la relation producteur-consommateur continue, de façon moderne, à être fondée sur un rapport de confiance et de proximité ? Il existe des initiatives de labellisation alternative ; c'est le cas de la Rede Ecovida (réseau Écovie), au Sud du Brésil, formée par des groupes d'agriculteurs écologiques, qui, en instituant son propre label, présente une proposition de surveillance et de contrôle participatif.

Au Brésil, il n'existe pas de subventions spécifiques pour les projets de conversion de l'agriculture conventionnelle en agriculture écologique, bien que certains organismes d'extension rurale incluent déjà des programmes d'aide aux agriculteurs. Partant du principe que l'agriculture écologique représente un changement de paradigme, les politiques de développement devraient non seulement inclure des mesures de subvention, des programmes de financement du changement technique, mais aller dans le sens d'une restructuration infrastructurelle et sociale dont

\footnotetext{
9 Au Brésil, il y a environ 20 entités de labellisation, dont les plus connues sont l'IBD, la fondation Mokiti Okada et ECOCERT.
} 
le but serait la construction d'un modèle de vie écologique. Dans ce sens, à l'initiative de quelques chercheurs, certaines institutions publiques ont déjà commencé à développer des programmes de recherche (universités et organismes de recherche en agronomie et en élevage).

L'institutionnalisation de l'agriculture écologique en tant qu'alternative différenciée de l'organisation de la production et de la consommation présente des conséquences pour la société et pour le mouvement social encore peu connues. Quoi qu'il en soit, l'institutionnalisation favorise un meilleur accès à des aliments de qualité, du point de vue écologique, ainsi qu'une plus grande participation des agriculteurs au processus de production. En s'instituant comme acteurs socioenvironnementaux, l'ensemble des acteurs sociaux, agriculteurs compris, développent des mesures de protection de la santé, de sécurité alimentaire, et renforcent des pratiques de préservation de l'environnement. Bien que le mouvement et la société y aient tout à gagner, des faits récents inquiètent certains militants, agriculteurs écologiques et experts. L'une des questions a trait à la possibilité ou non de maintenir le caractère alternatif du mouvement écologique : dans quelle mesure ou sous quelles formes institutionnelles l'agroécologie pourra-t-elle se développer en tant que proposition d'un paradigme alternatif par rapport au modèle industriel? Une agriculture agroécologique peut-elle se développer dans des structures qui opèrent selon les modèles de la rationalité marchande de l'agro-industrie? L'agroécologie va-t-elle se développer dans la direction d'un modèle industriel ou préservera-t-elle son caractère alternatif?

\section{Conclusion}

Si, d'un côté, le processus d'institutionnalisation de l'agriculture écologique dynamise la production et permet qu'un plus grand nombre de consommateurs ait accès à des produits de qualité écologiquement supérieure, de l'autre, il semble évident que la production écologique commence à absorber la logique de l'agriculture conventionnelle moderne. Pour avoir accès aux supermarchés, l'agriculture écologique se soumet aux contraintes de la consommation de masse, comme pratiquer des prix compétitifs, prendre soin de la présentation, offrir régulièrement des produits, etc. De ce fait, quelques-uns des principes de la production écologique semblent être abandonnés, par exemple lorsque celle-ci cesse d'être une agriculture de gestion obéissant aux principes de reproduction des systèmes naturels pour devenir une agriculture spécialisée, basée sur des apports minéraux et organiques. Dans ce sens, on pourrait se demander de quelle écologie on parle : d'une écologie aux bases naturelles ou d'une écologie aux intrants industrialisés et, par conséquent, d'une écologie industrielle? L'agriculture écologique s'engagera-t-elle sur la voie d'une production à modèle unique ou à modèles différenciés ?
Si l'on constate que l'agriculture écologique s'institutionnalise et que, par là, elle se transforme en s'adaptant au système du marché de masse, cela ne signifie pas l'abolition de la production agroécologique de commerce traditionnel et sélectif qui s'est instituée et propagée dans la deuxième phase du développement de l'agroécologie. Certains éléments signalent sa persistance à côté des autres modèles.

On observe également que, bien que l'agriculture biologique soit la plus structurée des agricultures alternatives, d'autres produits qui ne portent pas le label de la production écologique (agriculture de précision, intégrale) semblent conquérir le marché. C'est le fruit de l'impact de la production écologique sur l'agriculture. Cependant, l'affirmation de ces différents produits dépendra, d'un côté, de l'organisation des acteurs et, de l'autre, des critères, de la sensibilité et de la conscience écologique des consommateurs.

\section{Références}

Balestrin, N.L., 2002. Associação de Agricultura Organica do Paraná: uma proposta em (re)construção. Mestrado em Sociologia das Organizações, UFPR, Curitiba.

Bye, P., Schmidt, W., 2001. Agriculture familiale au Sud du Brésil ; d'une exclusion productiviste à une exclusion certifiée? Communication au XIX Congrès de la Société européenne pour la sociologie rurale, Dijon, 3-7 septembre.

César, C., 1999. De la conception du «naturel »: les catégories de l'entendement à l'œuvre chez les consommateurs de produits issus d'agriculture biologique. Contribution à une socioanthropologie de l'alimentation. Thèse de doctorat en sociologie, Université Paris X, Nanterre.

Guivant, J., 2003. Os supermercados na oferta de alimentos orgânicos : apelando aoestilo de vida ego-trip, Sociedade e Ambiente, VI, 2, 63-82.

Karam, K., 2001. Agricultura orgânica : estratégia para uma nova ruralidade na regiãometropolitana de Curitiba. Paraná. Doutorado em Meio Ambiente e Desenvolvimento, UFPR, Curitiba.

Ministério da Agricultura, Pecuária e Abastecimento (http//www.agricultura.gov.br).

Reed, M., 2001. Fight the future! How the contemporary campaigns of the UK organic movement have arisen from their composting of the past, Sociologia Ruralis, 41, 1, 131-145.

Rodrigues, R., 2001. Feira. Monografia, UFPR/Deciso, Curitiba.

Rucinski, J., 2000. Consumidores de alimentos orgânicos em Curitiba. Monografia de graduação, UFPR/Deciso, Curitiba.

Silva, P., Jr da, 2006. Escolhas e influências dos consumidores de alimentos na modernidade reflexiva: um estudo em Supermercados. Dissertação de Mestrado, UFPR, Curitiba.

Vogt, G., 2000. Entstehung und Entwicklung des ökologischen Landbaus im deutschsprachigen Raum, Bad Dürkheim, Söl.

Weid, J.M. von der, 1988. Projeto tecnologias alternativas/FASE, Proposta : experiência em educação popular, 36, 2-4. 